\title{
Appropriate Reconciliation of Cardiovascular Medications After Elective Surgery and Postdischarge Acute Hospital and Ambulatory Visits
}

\author{
Jonathan S. Lee, MD, MAS ${ }^{1 \star}$, Ralph Gonzales, MD, MSPH${ }^{1}$, Eric Vittinghoff, PhD², Kitty K. Corbett, PhD, MPH ${ }^{3}$, \\ Kirsten E. Fleischmann, MD', Neil Sehgal, PhD, MPH${ }^{5}$, Andrew D. Auerbach, MD, MPH ${ }^{5}$
}

\begin{abstract}
${ }^{1}$ Division of General Internal Medicine, University of California San Francisco, San Francisco, California; ${ }^{2}$ Department of Epidemiology and Biostatistics, University of California San Francisco, San Francisco, California; ${ }^{3}$ School of Public Health and Health Systems, University of Waterloo, Waterloo, Ontario, Canada; ${ }^{4}$ Division of Cardiology, University of California San Francisco, San Francisco, California; ${ }^{5}$ Division of Hospital Medicine, University of California San Francisco, San Francisco, California.
\end{abstract}

BACKGROUND: Few studies have assessed the quality and impact of postoperative cardiovascular medication reconciliation.

OBJECTIVE: To describe appropriate discharge reconciliation of cardiovascular medications and assess associations with postdischarge healthcare utilization in surgical patients.

DESIGN: Retrospective cohort study from January 2007 to December 2011.

SETTING: An academic medical center.

PATIENTS: Seven hundred and fifty-two adults undergoing elective noncardiac surgery and taking antiplatelet agents, beta-blockers, renin-angiotensin system inhibitors, or statin lipid-lowering agents before surgery.

MEASUREMENTS: Primary predictor: appropriate discharge reconciliation of preoperative cardiovascular medications (continuation without documented contraindications). Primary outcomes: acute hospital visits (emergency department visits or hospitalizations) and unplanned ambulatory visits (primary care or surgical) at 30 days after surgery.
RESULTS: Preoperative medications were appropriately reconciled in 436 (58.0\%) patients. For individual medications, appropriate discharge reconciliation occurred for 156 of the 327 patients on antiplatelet agents (47.7\%), 507 of the 624 patients on beta-blockers (81.3\%), 259 of the 361 patients on renin-angiotensin system inhibitors (71.8\%), and 302 of the 406 patients on statins (74.4\%). In multivariable analyses, appropriate reconciliation of all preoperative medications was not associated with acute hospital (adjusted odds ratio [AOR], 0.94; 95\% confidence interval [Cl], 0.63-1.41) or unplanned ambulatory visits (AOR, 1.48; 95\% Cl, 0.94-2.35). Appropriate reconciliation of statin therapy was associated with lower odds of acute hospital visits (AOR, 0.47; 95\% Cl, $0.26-0.85)$. There were no other statistically significant associations between appropriate reconciliation of individual medications and either outcome.

CONCLUSIONS: Although large gaps in appropriate discharge reconciliation of chronic cardiovascular medications were common in patients undergoing elective surgery, these gaps were not consistently associated with postdischarge acute hospital or ambulatory visits. Journal of Hospital Medicine 2017;12:723-730. (C 2017 Society of Hospital Medicine
Medication reconciliation at hospital discharge is a critical component of the posthospital transition of care. ${ }^{1}$ Effective reconciliation involves a clear process for documenting a current medication list, identifying and resolving discrepancies, and then documenting decisions and instructions around which medications should be continued, modified, or stopped. ${ }^{2}$ Existing studies ${ }^{3-5}$ suggest that medication discrepancies are common during hospital discharge transitions of care and lead to preventable adverse drug events, patient disability, and increased healthcare utilization following hospital discharge, including physician office visits, emer-

\footnotetext{
*Address for correspondence and reprint requests: Jonathan S. Lee, MD, University of California, San Francisco, 1545 Divisadero Street, $2^{\text {nd }}$ Floor, San Francisco, CA 94143-0320; Telephone: 415-353-7900; Fax: 415-353-2640; E-mail: jonathan.lee2@ucsf.edu.

Additional Supporting Information may be found in the online version of this article.
}

Received: November 15, 2016; Revised: March 11, 2017; Accepted: March 22, 2017

2017 Society of Hospital Medicine DOI 10.12788/jhm.2808 gency department (ED) visits, and hospitalizations. ${ }^{6-8}$

While the majority of studies of medication discrepancies have been conducted in general medical patients, few have examined how gaps in discharge medication reconciliation might affect surgical patients. ${ }^{9,10}$ Two prior studies ${ }^{9,10}$ suggest that medication discrepancies may occur more frequently for surgical patients, compared with medical patients, particularly discrepancies in reordering home medications postoperatively, raising patient safety concerns for more than 50 million patients hospitalized for surgery each year. ${ }^{11}$ In particular, little is known about the appropriate discharge reconciliation of chronic cardiovascular medications, such as beta-blockers, renin-angiotensin system inhibitors, and statins in surgical patients, despite perioperative practice guidelines recommending continuation or rapid reinitiation of these medications after noncardiac surgery. ${ }^{12}$ Problems with chronic cardiovascular medications have been implicated as major contributors to ED visits and hospitalizations for adverse drug events, ${ }^{13,14}$ further highlighting the importance of safe and appropriate management of these medications.

To better understand the current state and impact of post- 
TABLE 1. Characteristics of Patients Hospitalized for Elective Noncardiac Surgery by Appropriate Cardiovascular Medication Reconciliation at Discharge ${ }^{a}$

\begin{tabular}{|c|c|c|c|}
\hline \multirow[b]{2}{*}{ Patient Characteristics } & \multicolumn{3}{|c|}{ Discharge Medication Reconciliation } \\
\hline & $\begin{array}{l}\text { Appropriate } \\
\mathrm{N}=436\end{array}$ & $\begin{array}{l}\text { Inappropriate } \\
\qquad \mathrm{N}=316\end{array}$ & $P$ Values \\
\hline \multicolumn{4}{|l|}{ Demographics } \\
\hline Age $(y)$, mean (SD) & $61.6(11.8)$ & $61.4(12.0)$ & .76 \\
\hline White, non-Hispanic & $315(72.3)$ & $231(73.1)$ & \\
\hline Black, non-Hispanic & $28(6.4)$ & $23(7.3)$ & \\
\hline Hispanic & $36(8.3)$ & $22(7.0)$ & \\
\hline Other ${ }^{b}$ & $57(13.1)$ & $40(12.7)$ & \\
\hline Surgical service, n (\%) & $110(25.2)$ & $63(19.9)$ & $<.001$ \\
\hline Vascular surgery & $22(5.1)$ & $17(5.4)$ & \\
\hline Gynecology/oncology & $21(4.8)$ & $40(12.7)$ & \\
\hline Rena//liver transplant surgery & $14(3.2)$ & $8(2.5)$ & \\
\hline Cardiothoracic surgery & $9(2.1)$ & $2(0.6)$ & \\
\hline Plastic surgery & $6(1.4)$ & $8(2.5)$ & \\
\hline \multicolumn{4}{|l|}{ Otolaryngology } \\
\hline \multicolumn{4}{|l|}{ Surgical cardiovascular risk } \\
\hline Revised cardiac risk index score, n (\%) & & & .02 \\
\hline 0 & $282(64.7)$ & $167(52.9)$ & \\
\hline 1 & $110(25.2)$ & $113(35.8)$ & \\
\hline 2 & $34(7.8)$ & $27(8.5)$ & \\
\hline \multicolumn{4}{|l|}{ Individual risk index criteria, n (\%) } \\
\hline History of heart failure & $32(7.3)$ & $15(4.8)$ & .15 \\
\hline \multicolumn{4}{|l|}{ Preoperative medication use, $\mathrm{n}(\%)^{c}$} \\
\hline Antiplatelet agent & $122(28.0)$ & $205(64.9)$ & $<.001$ \\
\hline Beta-blocker & $371(85.1)$ & $253(80.1)$ & .07 \\
\hline Renin-angiotensin inhibitor & $184(42.2)$ & $177(56.0)$ & $<.001$ \\
\hline Statin & $211(48.4)$ & $195(61.7)$ & $<.001$ \\
\hline \multicolumn{3}{|l|}{ Number of cardiovascular medications } & $<.001$ \\
\hline 1 & $154(35.3)$ & $47(14.9)$ & \\
\hline 2 & $151(34.6)$ & 89 (28.2) & \\
\hline 3 & $92(21.1)$ & $115(36.4)$ & \\
\hline 4 & $39(8.9)$ & $65(20.6)$ & \\
\hline \multicolumn{4}{|c|}{$\begin{array}{l}\text { aAppropriate medication reconciliation at discharge for cardiovascular medications being taken in the preoperative period was defined as medical record documentation that the medication was being prescribed at discharge, or doc } \\
\text { mentation of a new contraindication to the medication during hospitalization if it was not prescribed. }\end{array}$} \\
\hline \multicolumn{4}{|c|}{ DOther race and ethnicity consisted of patients whose race was reported as Asian, Pacific Islander, Native American, or Alaskan Native, and patients with unreported race or ethnicity. } \\
\hline
\end{tabular}

operative discharge medication reconciliation of chronic cardiovascular medications in surgical patients, we examined (1) the appropriate discharge reconciliation of 4 cardiovas- cular medication classes, and (2) the associations between the appropriate discharge reconciliation of these medication classes and postdischarge acute hospital and ambulatory vis- 
TABLE 2. Frequency of Preoperative Cardiovascular Medication Use and Appropriate Medication Reconciliation at Discharge in Patients Hospitalized for Elective Noncardiac Surgery

\begin{tabular}{|c|c|c|}
\hline Cardiovascular Medication ${ }^{\mathrm{a}}$ & $\begin{array}{l}\text { Preoperative Frequency }(\mathrm{N}=752) \\
\mathrm{n}(\%)\end{array}$ & $\begin{array}{c}\text { Appropriate Reconciliation }{ }^{b} \\
n / \mathrm{N}(\%)\end{array}$ \\
\hline Antiplatelet agent & $327(43.5)$ & 156/327 (47.7) \\
\hline Beta-blocker & $624(83.0)$ & 507/624 (81.3) \\
\hline Renin-angiotensin system inhibitor & $361(48.0)$ & 259/361 (71.8) \\
\hline Statin & $406(54.0)$ & 302/406 (74.4) \\
\hline \multicolumn{3}{|l|}{ Number of cardiovascular medications } \\
\hline 1 & $201(26.7)$ & 154/201 (76.6) \\
\hline 2 & $240(31.9)$ & $151 / 240(62.9)$ \\
\hline 3 & $207(27.5)$ & 92/207 (44.4) \\
\hline 4 & $104(13.8)$ & 39/104 (37.5) \\
\hline
\end{tabular}

TABLE 3. Associations Between Appropriate Cardiovascular Medication Reconciliation at Discharge and ED Visits or Hospitalizations 30 Days After Surgery

\begin{tabular}{|c|c|c|c|}
\hline Medication Reconciliation & \multicolumn{3}{|c|}{ ED Visits or Hospitalizations 30 Days After Surgery $(N=679)$} \\
\hline Inappropriate & 66/295 (22.4) & .63 & Reference \\
\hline Appropriate & 80/384 (20.8) & & $0.94(0.63-1.41)$ \\
\hline \multicolumn{4}{|l|}{ By medication } \\
\hline Inappropriate & 32/161 (19.9) & .40 & Reference \\
\hline Appropriate & 33/138 (23.9) & & $1.11(0.61-2.03)$ \\
\hline \multicolumn{4}{|l|}{ Beta-blocker } \\
\hline Inappropriate & 25/106 (23.6) & .71 & Reference \\
\hline Appropriate & $100 / 456(21.9)$ & & $0.95(0.57-1.60)$ \\
\hline \multicolumn{4}{|l|}{ Renin-angiotensin system inhibitor } \\
\hline \multicolumn{4}{|l|}{ Statin } \\
\hline Inappropriate & $30 / 94(31.9)$ & .004 & Reference \\
\hline Appropriate & 50/279 (17.9) & & $0.47(0.26-0.85)$ \\
\hline
\end{tabular}

its in patients hospitalized for elective noncardiac surgery at an academic medical center.

\section{METHODS}

\section{Study Design and Patient Selection}

We performed a retrospective analysis of data collected as part of a cohort study of hospitalized surgical patients admitted between January 2007 and December 2011. The original study was designed to assess the impact of a social market- ing intervention on guideline-appropriate perioperative beta-blocker use in surgical patients. The study was conducted at 1 academic medical center that had 2 campuses with full noncardiac operative facilities and a 600-bed total capacity. Both sites had preoperative clinics, and patients were recruited by review of preoperative clinic records. Institutional review boards responsible for all sites approved the study.

For this analysis, we included adults (age $>18$ years) undergoing elective noncardiac surgery, who were expected 
TABLE 4. Associations Between Appropriate Cardiovascular Medication Reconciliation at Discharge and Unplanned Ambulatory Visits 30 Days After Surgery

Unplanned Ambulatory Visits 30 Days After Surgery $(\mathrm{N}=679)$

\begin{tabular}{|c|c|c|c|}
\hline Medication Reconciliation & $\mathrm{n} / \mathrm{N}(\%)$ & $P$ Values & $\operatorname{AOR}(95 \% \mathrm{Cl})^{\mathrm{a}}$ \\
\hline \multicolumn{4}{|l|}{ Complete vs incomplete } \\
\hline Inappropriate & 41/295 (13.9) & .13 & Reference \\
\hline Appropriate & 70/384 (18.2) & & $1.48(0.94-2.35)$ \\
\hline \multicolumn{4}{|l|}{ By Medication } \\
\hline \multicolumn{4}{|l|}{ Antiplatelet agent } \\
\hline Inappropriate & 22/161 (13.7) & .37 & Reference \\
\hline Appropriate & 24/138 (17.4) & & $1.25(0.58-2.68)$ \\
\hline \multicolumn{4}{|l|}{ Beta-blocker } \\
\hline Inappropriate & 17/106 (16.0) & .47 & Reference \\
\hline Appropriate & $87 / 456(19.1)$ & & $1.18(0.65-2.15)$ \\
\hline \multicolumn{4}{|l|}{ Renin-angiotensin system inhibitor } \\
\hline Inappropriate & 13/95 (13.7) & .37 & Reference \\
\hline Appropriate & $40 / 225(17.8)$ & & $1.32(0.65-2.68)$ \\
\hline \multicolumn{4}{|l|}{ Statin } \\
\hline Inappropriate & 14/94 (14.9) & .83 & Reference \\
\hline Appropriate & $39 / 279(14.0)$ & & $1.06(0.48-2.32)$ \\
\hline
\end{tabular}

to remain hospitalized for at least 1 day and were taking antiplatelet agents (aspirin, aspirin-dipyridamole, or clopidogrel), beta-blockers, renin-angiotensin system inhibitors (angiotensin-converting-enzyme inhibitors or angiotensin-receptor blockers), or statin lipid-lowering agents.

\section{Data Collection}

Data Sources. We collected data from a structured review of medical records as well as from discharge abstract information obtained from administrative data systems. Data regarding patient demographics (age, sex, and race/ethnicity), medical history, preoperative cardiovascular medications, surgical procedure and service, and attending surgeon were obtained from a medical record review of comprehensive preoperative clinic evaluations. Data regarding complications during hospitalization were obtained from medical record review and administrative data (Supplement for International Classification of Diseases, Ninth Revision codes). ${ }^{15}$ Research assistants abstracting data were trained by using a comprehensive reference manual providing specific criteria for classifying chart abstraction data. Research assistants also were directly observed during initial chart abstractions and underwent random chart validation audits by a senior investigator to ensure consistency. Any discrepancies in coding were resolved by consensus among senior investigators.

Definition of Key Predictor: Appropriate Reconciliation. We abstracted discharge medication lists from the electronic medical record. We defined the appropriate reconciliation of cardiovascular medications at discharge as documentation in discharge instructions, medication reconciliation tools, or discharge summaries that a preadmission cardiovascular medication was being continued at discharge, or, if the medication was not continued, documentation of a new contraindication to the medication or complication precluding its use during hospitalization. Medication continuity was considered appropriate if it was continued at discharge irrespective of changes in dosage. By using this measure for individual medications, we also assessed appropriate reconciliation as an "all-or-none" complete versus incomplete measure (appropriate reconciliation of all preoperative cardiovascular medication classes the patient was taking). ${ }^{16}$

Definition of Outcomes. Our coprimary outcomes were acute hospital visits (ED visits or hospitalizations) and unplanned ambulatory visits (primary care or surgical) at 30 days after surgery. Postoperative ambulatory visits that were not planned prior to surgery were defined as unplanned. Outcomes were ascertained by patient reports during follow-up telephone questionnaires administered by trained research staff and verified by medical record review.

Definition of Covariates. Using these data, we calculated a Revised Cardiac Risk Index (RCRI) score, ${ }^{17}$ which estimates the risk of perioperative cardiac complications in patients undergoing surgery. Through chart abstraction data supplemented by diagnosis codes from administrative data, we also constructed variables indicating occurrences of postoperative complications anytime during hospitalization that might pose contraindications to continuation of the 4 cardiovascular medication classes studied. For example, if a 
chart indicated that the patient had an acute rise in creatinine (elevation of baseline creatinine by $50 \%$ or absolute rise of $1 \mathrm{mg} / \mathrm{dL}$ in patients with baseline creatinine greater than $3 \mathrm{mg} / \mathrm{dL}$ ) during hospitalization and a preoperative renin-angiotensin system inhibitor was not prescribed at discharge, we would have considered discontinuation appropriate. Other complications we abstracted were hypotension (systolic blood pressure less than $90 \mathrm{mmHg}$ ) for beta-blockers and renin-angiotensin system inhibitors, bradycardia (heart rate less than $50 \mathrm{bpm}$ ) for beta-blockers, acute kidney injury (defined above) and hyperkalemia for renin-angiotensin system inhibitors, and bleeding (any site) for antiplatelet agents.

\section{Statistical Analysis}

We used $\chi^{2}$ and Kruskal-Wallis tests to compare baseline patient characteristics. To assess associations between appropriate medication reconciliation and patient outcomes, we used multilevel mixed-effects logistic regression to account for the clustering of patients by the attending surgeon. We adjusted for baseline patient demographics, surgical service, the number of baseline cardiovascular medications, and individual RCRI criteria. We constructed separate models for all-or-none appropriate reconciliation and for each individual medication class.

As a sensitivity analysis, we constructed similar models by using a simplified definition of appropriate reconciliation based entirely on medication continuity (continued or not continued at discharge) without taking potential contraindications during hospitalization into account. For complete versus incomplete reconciliation, we also constructed models with an interaction term between the number of baseline cardiovascular medications and appropriate medication reconciliation to test the hypothesis that inappropriate reconciliation would be more likely with an increasing number of preoperative cardiovascular medications. Because this interaction term was not statistically significant, we did not include it in the final models for ease of reporting and interpretability. We performed all statistical analyses using Stata 14 (StataCorp, LLC, College Station, Texas), and used 2 -sided statistical tests and a $P$ value of less than .05 to define statistical significance.

\section{RESULTS}

\section{Patient Characteristics}

A total of 849 patients were enrolled, of which 752 (88.6\%) were taking at least 1 of the specified cardiovascular medications in the preoperative period. Their mean age was 61.5 ; $50.9 \%$ were male, $72.6 \%$ were non-Hispanic white, and $89.4 \%$ had RCRI scores of 0 or 1 (Table 1 ). The majority $(63.8 \%)$ were undergoing general surgery, orthopedic surgery, or neurosurgery procedures. In the preoperative period, $327(43.5 \%)$ patients were taking antiplatelet agents, 624 $(83.0 \%)$ were taking beta-blockers, $361(48.0 \%)$ were taking renin-angiotensin system inhibitors, and 406 (54.0\%) were taking statins (Table 2). Among patients taking antiplatelet agents, $271(82.9 \%)$ were taking aspirin alone, 21 (6.4\%) were taking clopidogrel alone, and $35(10.7 \%)$ were taking dual antiplatelet therapy with aspirin and clopidogrel. Nearly three-quarters of the patients $(551,73.3 \%)$ were taking medications from 2 or more classes, and the proportion of patients with inappropriate reconciliation increased with the number of preoperative cardiovascular medications.

Patients with and without appropriate reconciliation of all preoperative cardiovascular medications were similar in age, sex, and race/ethnicity (Table 1). Patients with inappropriate reconciliation of at least 1 medication were more likely to be on the urology and renal/liver transplant surgical services, have higher RCRI scores, and be taking antiplatelet agents, statins, renin-angiotensin system inhibitors, and 3 or more cardiovascular medications in the preoperative period.

\section{Appropriate Medication Reconciliation}

Four hundred thirty-six patients $(58.0 \%)$ had their baseline cardiovascular medications appropriately reconciled. Among all patients with appropriately reconciled medications, $1(0.2 \%)$ had beta-blockers discontinued due to a documented episode of hypotension; 17 (3.9\%) had renin-angiotensin system inhibitors discontinued due to episodes of acute kidney injury, hypotension, or hyperkalemia; and 1 $(0.2 \%)$ had antiplatelet agents discontinued due to bleeding. For individual medications, appropriate reconciliation between the preoperative and discharge periods occurred for 156 of the 327 patients on antiplatelet agents $(47.7 \%), 507$ of the 624 patients on beta-blockers (81.3\%), 259 of the 361 patients on renin-angiotensin system inhibitors $(71.8 \%)$, and 302 of the 406 patients on statins (74.4\%; Table 2).

\section{Associations Between Medication Reconciliation and Outcomes}

Thirty-day outcome data on acute hospital visits were available for $679(90.3 \%)$ patients. Of these, $146(21.5 \%)$ were seen in the ED or were hospitalized, and $111(16.3 \%)$ were seen for an unplanned primary care or surgical outpatient visit at 30 days after surgery. Patients with incomplete outcome data were more likely to have complete medication reconciliation compared with those with complete outcome data $(71.2 \%$ vs $56.6 \%, P=0.02)$. As shown in Table 3 , the proportion of patients with 30-day acute hospital visits was nonstatistically significantly lower in patients with complete medication reconciliation $(20.8 \%$ vs $22.4 \%, P=0.63)$ and the appropriate reconciliation of beta-blockers $(21.9 \%$ vs $23.6 \%, P=0.71)$ and renin-angiotensin system inhibitors (19.6\% vs $20.0 \%, P=0.93$ ), and nonsignificantly higher with the appropriate reconciliation of antiplatelet agents ( $23.9 \%$ vs $19.9 \%, P=0.40$ ). Acute hospital visits were statistically significantly lower with the appropriate reconciliation of statins $(17.9 \%$ vs $31.9 \%, P=0.004)$.

In hierarchical multivariable models, complete appropriate medication reconciliation was not associated with acute hospital visits (adjusted odds ratio [AOR], 0.94; 95\% confidence interval [CI], 0.63-1.41). For individual medications, 
appropriate reconciliation of statins was associated with lower odds of unplanned hospital visits (AOR, 0.47; 95\% $\mathrm{CI}, 0.26-0.85$ ), but there were no statistically significant associations between appropriate reconciliation of antiplatelet agents, beta-blockers, or renin-angiotensin system inhibitors and hospital visits (Table 3). Similarly, the proportion of patients with 30-day unplanned ambulatory visits was not statistically different among patients with complete reconciliation or appropriate reconciliation of individual medications (Table 4). Adjusted analyses were consistent with the unadjusted point estimates and demonstrated no statistically significant associations.

\section{Sensitivity Analysis}

Overall, 430 (57.2\%) patients had complete cardiovascular medication continuity without considering potential contraindications during hospitalization. Associations between medication continuity and acute hospital and ambulatory visits were similar to the primary analyses.

\section{DISCUSSION}

In this study of 752 patients hospitalized for elective noncardiac surgery, we found significant gaps in the appropriate reconciliation of commonly prescribed cardiovascular medications, with inappropriate discontinuation ranging from $18.8 \%$ to $52.3 \%$ for individual medications. Unplanned postdischarge healthcare utilization was high, with acute hospital visits documented in $21.5 \%$ of patients and unplanned ambulatory visits in $16.3 \%$ at 30 days after surgery. However, medication reconciliation gaps were not consistently associated with ED visits, hospitalizations, or unplanned ambulatory visits.

Our finding of large gaps in postoperative medication reconciliation is consistent with existing studies of medication reconciliation in surgical patients. ${ }^{9,10,18}$ One study found medication discrepancies in $40.2 \%$ of postoperative patients receiving usual care and discrepancies judged to have the potential to cause harm (such as the omission of beta-blockers) in $29.9 \% .{ }^{9}$ Consistent with our findings, this study also found that most postoperative medication discrepancies were omissions in reordering home medications, though at a rate somewhat higher than those seen in medical patients at discharge. ${ }^{5}$ While hospitalization by itself increases the risk of unintentional discontinuation of chronic medications, ${ }^{3}$ our results, along with existing literature, suggest that the risk for omission of chronic medications is unacceptably high.

We also found significant variation in reconciliation among cardiovascular medications, with appropriate reconciliation occurring least frequently for antiplatelet agents and most frequently for beta-blockers. The low rates of appropriate reconciliation for antiplatelet agents may be attributable to deliberate withholding of antiplatelet therapy in the postoperative period based on clinical assessments of surgical bleeding risk in the absence of active bleeding. Perioperative management of antiplatelet agents for non- cardiac surgery remains an unclear and controversial topic, which may also contribute to the variation noted. ${ }^{19}$ Conversely, beta-blockers demonstrated high rates of preoperative use (over $80 \%$ of patients) and appropriate reconciliation. Both findings are likely attributable in part to the timing of the study, which began prior to the publication of the Perioperative Ischemic Evaluation trial, which more definitively demonstrated the potential harms of perioperative beta-blocker therapy. ${ }^{20}$

Despite a high proportion of patients with discontinuous medications at discharge, we found no associations between the appropriate reconciliation of beta-blockers, renin-angiotensin system inhibitors, and antiplatelet agents and acute hospital or ambulatory visits in the first 30 days after discharge. One explanation for this discrepancy is that, although we focused on cardiovascular medications commonly implicated in acute hospital visits, the vast majority of patients in our study had low perioperative cardiovascular risk as assessed by the RCRI. Previous studies have demonstrated that the benefit of perioperative beta-blocker therapy is predominantly in patients with moderate to high perioperative cardiovascular risk. ${ }^{21,22}$ It is possible that the detrimental effects of the discontinuation of chronic cardiovascular medications are more prominent in populations at a higher risk of perioperative cardiovascular complications or that complications will occur later than 30 days after discharge. Similarly, while the benefits of continuation of renin-angiotensin system inhibitors are less clear, ${ }^{23}$ few patients in our cohort had a history of congestive heart failure $(6.3 \%)$ or coronary artery disease (13.0\%), 2 conditions in which the impact of perioperative discontinuation of renin-angiotensin inhibitor or beta-blocker therapy would likely be more pronounced. ${ }^{24,25}$ An additional explanation for the lack of associations is that, while multiple studies have demonstrated that medication errors are common, the proportion of errors with the potential for harm is much lower, and the proportion that causes actual harm is lower still., ${ }^{5,26,27}$ Thus, while we likely captured high-severity medication errors leading to acute hospital or unplanned ambulatory visits, we would not have captured medication errors with lower severity clinical consequences that did not result in medical encounters.

We did find an association between the continuation of statin therapy and reduced ED visits and hospitalizations. This finding is supported by previous studies of patients undergoing noncardiac surgery, including 1 demonstrating an association between immediate postoperative statin therapy and reduced in-hospital mortality ${ }^{28}$ and another study demonstrating an association between postoperative statin therapy and reductions in a composite endpoint of 30-day mortality, atrial fibrillation, and nonfatal myocardial infarction..$^{29}$ Alternatively, this finding could reflect the effects of unaddressed confounding by factors contributing to statin discontinuation and poor health outcomes leading to acute hospital visits, such as acute elevations in liver enzymes.

Our study has important implications for patients undergoing elective noncardiac surgery and the healthcare providers 
caring for them. First, inappropriate omissions of chronic cardiovascular medications at discharge are common; clinicians should increase their general awareness and focus on appropriately reconciling these medications, for even if our results do not connect medication discontinuity to readmissions or unexpected clinical encounters, their impact on patients' understanding of their medications remains a potential concern. Second, the overall high rates of unplanned postdischarge healthcare utilization in this study highlight the need for close postdischarge monitoring of patients undergoing elective surgical procedures and for further research to identify preventable etiologies of postdischarge healthcare utilization in this population. Third, further study is needed to identify specific patient populations and medication classes, in which appropriate reconciliation is associated with patient outcomes that may benefit from more intensive discharge medication reconciliation interventions.

Our study has limitations. First, the majority of patients in this single-center study were at low risk of perioperative cardiovascular events, and our results may not be generalizable to higher-risk patients undergoing elective surgery. Second, discharge reconciliation was based on documentation of medication reconciliation and not on patient-reported medication adherence. In addition, the ability to judge the accuracy of discharge medication reconciliation is in part dependent on the accuracy of the admission medication reconciliation. Thus, although we used preoperative medication regimens documented during preadmission visits to comprehensive preoperative clinics for comparison, discrepancies in these preoperative regimens could have affected our analysis of appropriate discharge reconciliation. Third, inadequate documentation of clinical reasons for discontinuing medications may have led to residual confounding by indication in our observational study. Finally, the outcomes available to us may have been relatively insensitive to other adverse effects of medication discontinuity, such as patient symptoms (eg, angina severity), patient awareness of medications, or work placed on primary care physicians needing to "clean up" erroneous medication lists.

In conclusion, gaps in appropriate discharge reconciliation of chronic cardiovascular medications were common but not consistently associated with postdischarge acute hospital or unplanned ambulatory visits in a relatively low-risk cohort of patients undergoing elective surgery. While appropriate medication reconciliation should always be a priority, further study is needed to identify medication reconciliation approaches associated with postdischarge healthcare utilization and other patient outcomes.

Disclosure: Dr. Lee reports receiving grant support from the Health Resources and Services Administration (T32HP19025). Dr. Vittinghoff reports receiving grant support from the Agency for Healthcare Research and Quality. Dr. Auerbach and Dr. Fleischmann report receiving grant support from the National Institutes of Health. Dr. Auerbach also reports receiving honorarium as Editor-in-Chief of the Journal of Hospital Medicine. Dr. Corbett reports receiving grant and travel support from Simon Fraser University. The remaining authors have no disclosures to report.

\section{References}

1. The Joint Commission. National Patient Safety Goals. 2016; https://www.jointcommission.org/standards_information/npsgs.aspx. Accessed June 21, 2016.

2. Institute for Healthcare Improvement. Medication Reconciliation to Prevent Adverse Drug Events. 2016; http://www.ihi.org/topics/ADEsMedicationReconciliation/Pages/default.aspx. Accessed June 24, 2016.

3. Bell CM, Brener SS, Gunraj N, et al. Association of ICU or hospital admission with unintentional discontinuation of medications for chronic diseases. JAMA. 2011;306(8):840-847.

4. Coleman EA, Smith JD, Raha D, Min SJ. Posthospital medication discrepancies: prevalence and contributing factors. Arch Intern Med. 2005;165(16):1842-1847.

5. Wong JD, Bajcar JM, Wong GG, et al. Medication reconciliation at hospital discharge: evaluating discrepancies. Ann Pharmacother. 2008;42(10):1373-1379.

6. Forster AJ, Clark HD, Menard A, et al. Adverse events among medical patients after discharge from hospital. CMAJ. 2004;170(3):345-349.

7. Forster AJ, Murff HJ, Peterson JF, Gandhi TK, Bates DW. The incidence and severity of adverse events affecting patients after discharge from the hospital. Ann Intern Med. 2003;138(3):161-167.

8. Forster AJ, Murff HJ, Peterson JF, Gandhi TK, Bates DW. Adverse drug events occurring following hospital discharge. JGIM. 2005;20(4):317-323.

9. Kwan Y, Fernandes OA, Nagge JJ, et al. Pharmacist medication assessments in a surgical preadmission clinic. Arch Intern Med. 2007;167(10):1034-1040.

10. Unroe KT, Pfeiffenberger T, Riegelhaupt S, Jastrzembski J, Lokhnygina Y, Colon-Emeric C. Inpatient Medication Reconciliation at Admission and Discharge: A Retrospective Cohort Study of Age and Other Risk Factors for Medication Discrepancies. Am J Geriatr Pharmacother. 2010;8(2):115-126.

11. CDC - National Center for Health Statistics. Fast Stats: Inpatient Surgery. http:// www.cdc.gov/nchs/fastats/inpatient-surgery.htm. Accessed on June 24, 2016.

12. Fleisher LA, Fleischmann KE, Auerbach AD, et al. 2014 ACC/AHA guideline on perioperative cardiovascular evaluation and management of patients undergoing noncardiac surgery: a report of the American College of Cardiology/ American Heart Association Task Force on Practice Guidelines. Circulation. 2014;130(24):e278-e333.

13. Budnitz DS, Lovegrove MC, Shehab N, Richards CL. Emergency hospitalizations for adverse drug events in older Americans. N Engl J Med. 2011;365(21):20022012.

14. Budnitz DS, Pollock DA, Weidenbach KN, Mendelsohn AB, Schroeder TJ, Annest JL. National surveillance of emergency department visits for outpatient adverse drug events. JAMA. 2006;296(15):1858-1866.

15. Bozic KJ, Maselli J, Pekow PS, Lindenauer PK, Vail TP, Auerbach AD. The influence of procedure volumes and standardization of care on quality and efficiency in total joint replacement surgery. J Bone Joint Surg Am. 2010;92(16):2643-2652.

16. Nolan T, Berwick DM. All-or-none measurement raises the bar on performance. JAMA. 2006;295(10):1168-1170.

17. Lee TH, Marcantonio ER, Mangione CM, et al. Derivation and prospective validation of a simple index for prediction of cardiac risk of major noncardiac surgery. Circulation. 1999;100(10):1043-1049.

18. Gonzalez-Garcia L, Salmeron-Garcia A, Garcia-Lirola MA, Moya-Roldan S, Belda-Rustarazo S, Cabeza-Barrera J. Medication reconciliation at admission to surgical departments. J Eval Clin Pract. 2016;22(1):20-25.

19. Devereaux PJ, Mrkobrada M, Sessler DI, et al. Aspirin in patients undergoing noncardiac surgery. N Engl J Med. 2014;370(16):1494-1503.

20. Devereaux PJ, Yang H, Yusuf S, et al. Effects of extended-release metoprolol succinate in patients undergoing non-cardiac surgery (POISE trial): a randomised controlled trial. Lancet. 2008;371(9627):1839-1847.

21. Lindenauer PK, Pekow P, Wang K, Mamidi DK, Gutierrez B, Benjamin EM. Perioperative beta-blocker therapy and mortality after major noncardiac surgery. N Engl J Med. 2005;353(4):349-361.

22. London MJ, Hur K, Schwartz GG, Henderson WG. Association of perioperative beta-blockade with mortality and cardiovascular morbidity following major noncardiac surgery. JAMA. 2013;309(16):1704-1713

23. Rosenman DJ, McDonald FS, Ebbert JO, Erwin PJ, LaBella M, Montori VM. Clinical consequences of withholding versus administering renin-angiotensin-aldosterone system antagonists in the preoperative period. J Hosp Med. 2008;3(4):319-325.

24. Andersson C, Merie C, Jorgensen M, et al. Association of beta-blocker therapy with risks of adverse cardiovascular events and deaths in patients with ischemic heart disease undergoing noncardiac surgery: a Danish nationwide cohort study. JAMA Intern Med. 2014;174(3):336-344

25. Yancy CW, Jessup M, Bozkurt B, et al. 2013 ACCF/AHA Guideline for the Management of Heart Failure A Report of the American College of Cardiology Foundation/American Heart Association Task Force on Practice Guidelines. Circulation. 2013;128(16):E240-E327

26. Kwan JL, Lo L, Sampson M, Shojania KG. Medication reconciliation during transitions of care as a patient safety strategy: a systematic review. Ann Intern Med. 
2013;158(5 Pt 2):397-403.

27. Tam VC, Knowles SR, Cornish PL, Fine N, Marchesano R, Etchells EE. Frequency, type and clinical importance of medication history errors at admission to hospital: a systematic review. CMAJ. 2005;173(5):510-515.

28. Lindenauer PK, Pekow P, Wang K, Gutierrez B, Benjamin EM. Lipid-lowering therapy and in-hospital mortality following major noncardiac surgery. JAMA. 2004;291(17):2092-2099.

29. Raju MG, Pachika A, Punnam SR, et al. Statin Therapy in the Reduction of Cardiovascular Events in Patients Undergoing Intermediate-Risk Noncardiac, Nonvascular Surgery. Clin Cardiol. 2013;36(8):456-461. 